\title{
Mutagenesis and Gene Cloning in Schizosaccharo- myces pombe Using Nonhomologous Plasmid Integration and Rescue
}

\author{
Charles S. Hoffman and \\ Robert Welton \\ Boston College, Chestnut Hill, \\ MA, USA
}

\begin{abstract}
Genes are commonly cloned in yeasts and bacteria by plasmid complementation, where the introduction of the gene of interest into a host strain carrying a recessive mutation in that gene suppresses the host's mutant phenotype. However, a lack of low copy cloning vectors in the fission yeast Schizosaccharomyces pombe can complicate this approach especially when overexpression of one gene may suppress a defect in another gene or when overexpression of the desired gene is detrimental, if not lethal, to the cell. We describe here a method of identifying mutations in $\mathrm{S}$. pombe that allows for the rapid and direct cloning of the defective gene. This involves the nonhomologous integration of a marked plasmid into the yeast genome and its subsequent rescue into Escherichia coli, so that DNA at the site of insertion is incorporated into the recovered plasmid. As two of three insertions obtained in this study occurred outside of the affected gene's open reading frame, this method should be applicable to cloning both essential genes and nonessential genes.
\end{abstract}

\section{INTRODUCTION}

The budding yeast Saccharomyces cerevisiae and the fission yeast Schizosaccharomyces pombe are important eukaryotic model organisms for the study of many fundamental biological processes such as cell cycle control, transcriptional and translational regulation and signal transduction $(5,11,13)$. This is largely due to three characteristics of these yeasts. First, they are single-cell organisms that can be maintained in a haploid state, allowing identification of rare mutants that carry either dominant or recessive mutant alleles that affect the process under study. Second, controlled mating of haploid strains permits the construction of diploid strains, which can then sporulate to produce asci containing four haploid spores. This sexual life cycle is exploited to provide important genetic information about mutant alleles with regard to dominance/recessiveness, complementation, linkage and epistatic relationships. Finally, yeast can be transformed with autonomously replicating plasmids to allow for cloning of genes based on their ability to confer an identifiable phenotype. Wild-type alleles of genes are frequently cloned by screening a plasmid library for clones that confer the wild-type phenotype upon a recessive mutant host strain. One can also clone a dominant mutant allele by screening a library that carries this allele for plasmids that can confer the mutant phenotype upon a wild-type host strain. Genes can also be cloned as multicopy or high copy suppressors. In this case, the overexpression of the cloned gene suppresses a defect in a different gene in the host.

Cloning in S. cerevisiae has been greatly facilitated by the construction of libraries in plasmid vectors containing centromeres that maintain a low copy number, but a high fidelity of mitotic transmission (14). This is technically possible due to the small size of the $S$. cerevisiae centromere that is less than 200 bp (2). While $S$. pombe can also maintain autonomous plasmids, there are no low copy mitotically stable plasmids available. This is because $S$. pombe centromeres are greater than 40 kbp (2), making them too large to incorporate into cloning vectors. Thus, $S$. pombe transformants generally carry plasmids in moderate to high copy number (6).

There are two potential problems in cloning $S$. pombe genes related to the lack of low copy number plasmids. First, clones carrying multicopy suppressors can suppress the mutant phenotype of a host strain as well as, if not better than, clones carrying a wild-type allele of the gene defective in the host. While multicopy suppression is unlikely to occur in the cloning of genes in biosynthetic pathways, it is common in signal transduction or transcriptional regulation studies where multiple pathways can control a single biological process. Second, genes that are detrimental to the host when overexpressed may be resistant to cloning since the desired transformants may grow very poorly, if at all.

We describe a strategy for cloning $S$. 
pombe genes that may otherwise be resistant to cloning by plasmid complementation. This strategy involves insertional mutagenesis by a marked plasmid followed by rescue of the plasmid, along with genomic DNA from the site of insertion, into Escherichia coli. As sequence determination of the $S$. pombe genome is almost completed, DNA sequence information of sites of integration will be sufficient to allow for the immediate identification of genes of interest and their cloning using PCR technology. Therefore, unlike plasmid complementation, genes can be cloned by this approach without ever needing to be overexpressed in $S$. pombe.

\section{MATERIALS AND METHODS}

\section{Insertional Mutagenesis}

The $S$. pombe strain CHP578 $\left(h^{+}\right.$ fbp1::ura4+ ura4::fbpl-lacZ leu1-32 his3-Dl) was used for insertional mutagenesis. This strain carries two integrated reporter genes ( $S$. pombe ura 4 and $E$. coli lacZ) fused to the $f b p l$ promoter that is transcriptionally repressed by glucose (8). It also carries the his3-Dl deletion allele (1). Yeast cells were transformed to $\mathrm{His}^{+}$as previously described (4) with $1 \mu \mathrm{g}$ NgoMIV-linearized (New England Biolabs, Beverly, MA, USA) plasmid pAF1 which carries the his ${ }^{+}$gene (Reference 12 and Figure 1). The transformed cells were plated to PM medium (16) lacking histidine (PM-his) to select for cells that received the pAF1 DNA.

\section{Mutant Screen}

Mutations that inhibit the PKA activation pathway cause both the derepression of $f b p l$ transcription $(8,9)$ and the derepression of gluconate uptake (3) and are designated as conferring a glucose insensitive transcription ( $\mathrm{Git}^{-}$) phenotype. We therefore screened for mutations in this pathway by replica plating from the PM-his transformation plates to SC-ura (15) (containing $8 \%$ glucose to screen for constitutive $f b p l$ $\mathrm{ura}^{+}$expression) and to synthetic complete (SC) with $3 \%$ gluconate as the carbon source (to screen for constitutive gluconate uptake that abolishes a lag in growth when shifting from glucose- to gluconate-based media) (3). Colonies that grew both on SC-ura within two days and on SC gluconate within three days were purified and assayed for $\beta$-galactosidase ( $\beta$-gal) activity expressed from the fbpl-lacZ reporter in cells grown under glucoserich conditions $(8)$. Those strains with elevated $\beta$-gal activity were crossed to strain CHP555 $\left(h^{-}\right.$fbpl::ura4 ${ }^{+}$ ura4::fbpl-lacZ ade6-M216 lys 1-131 leul-32 his3-D1) to see if the His ${ }^{+}$phenotype cosegregated with the Git- phenotype. Three mutants were identified this way and were used to rescue the integrated plasmids along with flanking genomic DNA as described below.

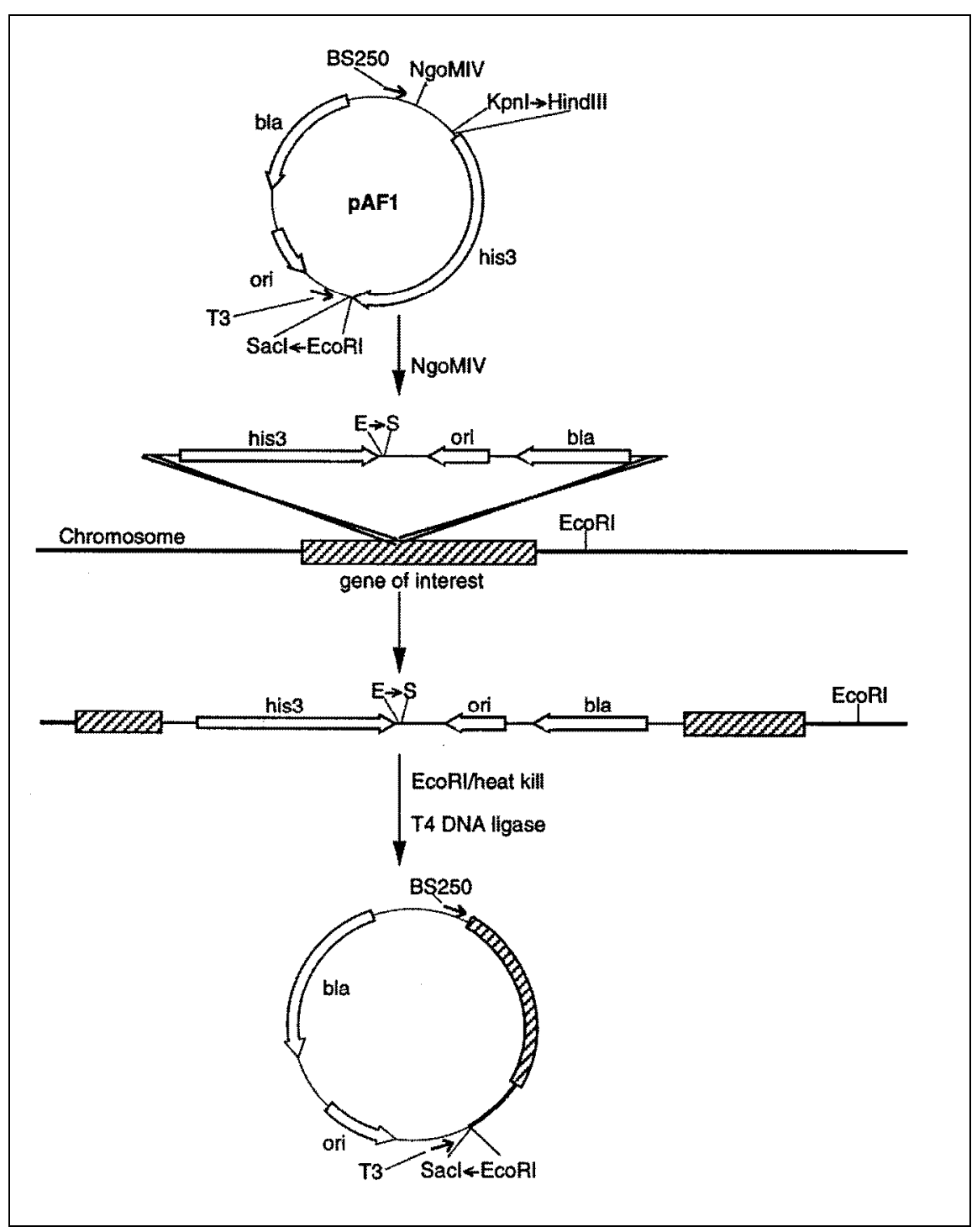

Figure 1. Schematic diagram of the plasmid insertion and rescue strategy. Plasmid pAF1 carries the his3 gene cloned into the EcoRV site of pBluescript SK+ (12). Other key features to note include a unique $N g o$ MI site, the SacI to EcoRI ( $<<-E)$ polylinker $3^{\prime}$ to his3 and the sites to which sequencing primers BS250 and T3 anneal. Plasmid pAF1 is linearized with NgoMIV and used to transform a his3Dl strain to $\mathrm{His}^{+}$. Nonhomologous insertion of the plasmid into a gene of interest will result in the desired mutant phenotype. Once the insertion event has been shown to be linked to the mutant phenotype (all mutant progeny are $\mathrm{His}^{+}$in crosses of the integrant with a his3-D1 tester strain), genomic DNA can be isolated, digested with a restriction enzyme that cuts in the SacI to EcoRI polylinker (in this case $E c o$ RI) and circularized by ligation to create a plasmid with most of the pBluescript SK+ backbone and a portion of the genomic DNA from the site of integration. The ends of the cloned genomic DNA can be sequenced with primers BS250 (reading across the insertion junction) and T3 (reading from the polylinker across the restriction site in the flanking DNA). 


\section{Plasmid Rescue into E. coli}

Plasmids carrying genomic DNA from the site of integration were rescued into $E$. coli by isolating genomic DNA from the integrants, digesting the DNA with a restriction enzyme, ligating the DNA and transforming $E$. coli to ampicillin-resistance (Figure 1). All restriction enzymes and T4 DNA ligase were purchased from New England Biolabs and used according to the manufacturer's instructions. Genomic DNA was prepared from $10 \mathrm{~mL}$ yeast extract liquid (YEL) cultures (5) by Smash and Grab as previously described (7) (as done for preparation of genomic DNA for Southern hybridization) and resuspended in $100 \mu \mathrm{L} \mathrm{H}_{2} \mathrm{O}$. Restriction digestion of this DNA was carried out using $5 \mu \mathrm{L}$ DNA in a $50 \mu \mathrm{L}$ reaction mixture with $40 \mathrm{U}$ enzyme for $1 \mathrm{~h}$. For $E c o$ RI or $X b a \mathrm{I}$ digests, the enzyme was heat inactivated at $80^{\circ} \mathrm{C}$ for $20 \mathrm{~min}$.

Following enzyme inactivation, 2.5 $\mu \mathrm{L} 20 \mathrm{mmol} / \mathrm{L}$ ATP and $0.5 \mu \mathrm{L}$ T4 DNA ligase were added to promote circularization of the DNA molecules. Ligation reactions were incubated at $16^{\circ} \mathrm{C}$ for $1 \mathrm{~h}$, ethanol precipitated and resuspended in $5 \mu \mathrm{L}$ water. Electroporation-competent $E$. coli strain XL1Blue (Stratagene, La Jolla, CA, USA) was transformed according to the manufacturer's instructions with $1 \mu \mathrm{L}$ of the precipitated ligation reaction. For genomic DNA digests with enzymes other than EcoRI or XbaI, phenol-chloroform extraction was used to inactivate the enzyme. The digestions were then ethanol precipitated and resuspended in a $50 \mu \mathrm{L}$ ligation mixture. Ligation, ethanol precipitation and transformation of E. coli were carried out as described above.

\section{DNA Sequence Analyses}

DNA sequencing was performed on rescued plasmids with primer BS250 5'-TTTTTGGGGTCGAGGTGC-3' that sequences across the insertion junction and with the T3 promoter primer $5^{\prime}$ ATTAACCCCTCACTAAAGGGA-3' that sequences across the restriction site cut in the flanking genomic region (Figure 1). DNA sequencing was performed using Big Dye ${ }^{\mathrm{TM}}$ Terminators (PE Biosystems, Foster City, CA,
USA) on an ABI 377XL Automated Sequencer by Bioserve Biotechnologies (Laurel, MD, USA).

\section{RESULTS AND DISCUSSION}

\section{Overview of Insertional Mutagenesis and Gene Cloning Strategy}

The goal of this study was to determine whether or not we could generate untargeted mutations in $S$. pombe genes by the nonhomologous integration of a plasmid, and then rescue the plasmid along with some of the flanking genomic DNA back into $E$. coli for DNA sequence analysis. A similar approach has been developed using a PCR product carrying the $\mathrm{ura}^{+}$gene with rescue by inverse PCR (P.G. Young, personal communication). We conducted this study since we already use $\mathrm{rra}^{+}$as a reporter for $f b p l$ transcription (see Materials and Methods). We also hoped to develop a method for cloning DNA from the site of insertion that would be simpler than inverse PCR and would allow for the rescue of relatively large $(>6 \mathrm{~kb})$ portions of genomic DNA directly as cloned DNA.

Our general strategy is outlined in Figure 1. Plasmid pAF1, carrying the $S$. pombe his $3^{+}$gene (12), was linearized and used to transform the host strain to His $^{+}$. The S. pombe strain CHP578 carries a complete deletion of his 3 to prevent homologous plasmid integration at the his 3 locus or gene conversion of the his 3 mutant allele to his $3^{+}$. These transformants were then screened for the desired mutant phenotype. Candidates were tested by a linkage analysis to see if the His ${ }^{+}$phenotype was linked to the desired mutant phenotype, which indicated that the plasmid insertion was responsible for the mutant phenotype.

Genomic DNA was isolated from the mutant transformant and digested with a restriction enzyme that cuts in the polylinker between the his $3^{+}$gene and the pBluescript ${ }^{\circledR}$ II SK+ (Stratagene, La Jolla, CA, USA) backbone of pAF1 and in the flanking genomic DNA. This DNA was circularized by ligation and used to transform $E$. coli to ampicillinresistance. Finally, the two ends of the insert DNA were sequenced and the sequence was analyzed by Blast search of the GenBank ${ }^{\circledR}$ DNA database and the $S$. pombe genome database (http://www. sanger.ac.uk/Projects/S_pombe/blast_ server.shtml) to identify the gene at the site of insertion.

\section{Insertional Mutagenesis and Mutant Screen}

For this study, we linearized pAF1 at the unique $N g$ oMIV site over $300 \mathrm{bp}$ from the his $3^{+}$gene to avoid loss of the his 3 function, should DNA be removed from the ends of the molecule before or during the integration process. His ${ }^{+}$ transformants were screened for a mutant phenotype, in this case the coordinate increase of fbpl-ura 4 transcription and gluconate uptake that is characteristic of strains carrying mutations in git genes involved in the activation of protein kinase $\mathrm{A}$ (PKA) in response to glucose detection $(8,9)$. From a total of eight transformations, we screened approximately 6800 medium-sized to large His ${ }^{+}$colonies (see Materials and Methods). There was a significant background of unstable $\mathrm{His}^{+}$microcolonies (10-20× the number of larger colonies) on the transformation plates, most likely due to uptake but not integration of pAF1. However, we made no effort to clean up this background since these colonies did not interfere with the detection of the desired mutants.

We identified three colonies that appeared to be git mutants based on the following criteria (see Materials and Methods). These strains grow well on SC-ura medium containing $8 \%$ glucose, which indicates a possible defect in glucose repression of transcription of the fbpl-ura4+ reporter. The strains grow well when transferred from a glucose-based medium to a gluconatebased medium, indicating a defect in glucose repression of gluconate uptake (3). Also, the strains express elevated levels of $\beta$-gal activity in cells grown in a glucose-rich medium, which shows a defect in glucose repression of $f b p l$ lacZ transcription (data not shown). The phenotypes co-segregate with the $\mathrm{His}^{+}$phenotype in crosses involving these strains (in 3-12 tetrads examined for each cross, two $\mathrm{His}^{+} \mathrm{Git}^{-}$progeny and two $\mathrm{His}^{-} \mathrm{Git}^{+}$progeny were observed), indicating that the integrated plasmid is responsible for the $\mathrm{Git}^{-}$ 


\section{Research Report}

Table 1. Plasmid Rescue as a Function of Restriction Digests of Genomic DNA

\begin{tabular}{|c|c|c|c|}
\hline Integrant & Restriction Enzyme & $\begin{array}{c}\text { Number of } \\
\text { Transformants }\end{array}$ & $\begin{array}{c}\text { Cloned Flanking } \\
\text { DNA (kb) }\end{array}$ \\
\hline CHP606 & EcoRl & 80 & 6.1 \\
\hline \multirow[t]{4}{*}{ CHP616 } & EcoRI & 1600 & 0.9 \\
\hline & Sacl & 30 & 0.9 \\
\hline & Sacll & 1 & 4.2 \\
\hline & Smal-BsaBla & 1 & 0.15 \\
\hline \multirow[t]{2}{*}{ CHP617 } & EcoRI & 1000 & 0.8 \\
\hline & Xbal & 300 & 2.3 \\
\hline \multicolumn{4}{|c|}{$\begin{array}{l}\text { Plasmids were rescued into } E \text {. coli from each of the three integrant strains after } \\
\text { restriction digestion of genomic DNA and ligation (see Materials and Methods). } \\
\text { Approximate numbers of transformants and the size of the cloned genomic DNA } \\
\text { are presented. } \\
\text { a Smal, which cuts in the plasmid polylinker, and BsaBI, which cuts in the flanking } \\
\text { genomic DNA, produce blunt-ends that are compatible for ligation. }\end{array}$} \\
\hline
\end{tabular}

mutant phenotype. This portion of the work demonstrates our ability to identify insertion mutations by plasmid integration.

\section{Rescue of Integrated Plasmids}

The next step was to rescue the integrated plasmid along with some of the genomic DNA from the site of integration into E. coli. We isolated genomic DNA from integrant strains and digested it with a restriction enzyme that cuts the plasmid in the $S a c$ I to EcoRI portion of the polylinker between his $3^{+}$ and the majority of the pBluescript II $\mathrm{SK}+$ backbone containing the origin of replication and bla selectable marker for transformation of E. coli (Figure 1). After inactivation of the restriction enzyme, the DNA fragments were circularized by ligation and used to transform $E$. coli to ampicillin-resistance.

As shown in Table 1, we were able to 
Table 2. Sequence Information from Rescued Plasmids

\begin{tabular}{|lcccc|}
\hline Integrant & Gene & $\begin{array}{c}\text { Site of } \\
\text { Insertion }\end{array}$ & $\begin{array}{c}\text { Genomic } \\
\text { Sequence }\end{array}$ & $\begin{array}{c}\text { Additional Sequence } \\
\text { at Insertion }\end{array}$ \\
\hline CHP606 & git5 & -263 & ACCATTA CTATTCT & none \\
CHP616 & git3 & +502 & ACAAATG TATTTCT & his3 bp 941 to 674 \\
CHP617 & $p k a 1$ & -155 & TATGTAT ATAAACC & none \\
DNA sequence analyses of the rescued plasmids using primers BS250 and T3 \\
(see Materials and Methods) were used to determine the genes altered by plas- \\
mid insertion, the sites of insertion relative to the translational start sites and the \\
presence of an additional 268 bp of the his3 gene (accession number L19524) in \\
CHP616. The sequence at the site of insertion is given as the proximal seven \\
bases of the two sequences flanking the inserted plasmid. The right-most se- \\
quence was determined by sequence analysis of the rescued plasmids using \\
primer BS250. The left-most sequence is inferred from the wild-type genomic se- \\
quence. However, it is not known if this sequence is intact in the integrant strains \\
since the plasmid recovery does not lead to rescue of this insertion junction (see \\
Figure 1). Accession numbers for the sequence entries used in determining in- \\
sertion sites relative to translational start sites are AB004535 (orf3; git5+), \\
AF085162 (git3 ${ }^{+}$) and D23667 (pka1+).
\end{tabular}

generate $E$. coli transformants using several different restriction enzymes that allowed for the cloning of varying amounts of the flanking genomic DNA. The protocol is notable for its simplicity and brevity (see Materials and Methods). However, it is possible that any given integration event may occur at too great a distance from the nearest $E c o$ RI site in the genome to allow for rescue.

Many enzymes (SacI, SacII, NotI, EagI, XbaI, SpeI, BamHI, SmaI, PstI or $E c o$ RI) can be used for plasmid rescue. We strongly recommend that initial at- tempts should be made with either EcoRI or XbaI because they can be heat inactivated and digest yeast DNA more frequently than do most of the other enzymes amenable to this procedure. In addition, rescue attempts with EcoRI were very efficient, even when the recovered genomic DNA was $6.1 \mathrm{~kb}$ (Table 1). Although SmaI digestion produces a blunt end that can be used in combination with other blunt-end cutters (as was done with $B s a \mathrm{BI}$; see Table 1 ), the fact that only one transformant was obtained this way, even though the

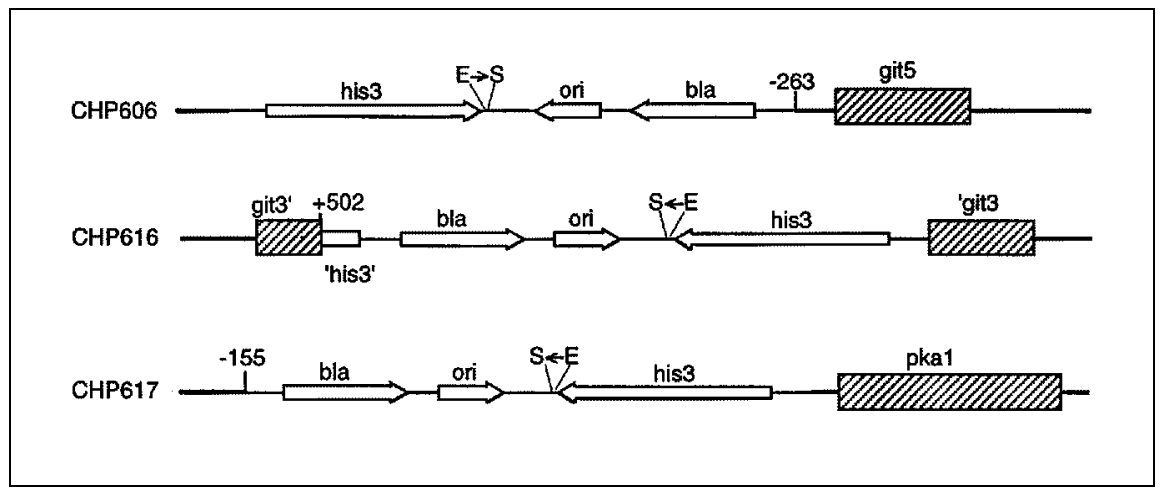

Figure 2. Schematic diagram of the insertions in three git mutant integrants. The position and orientation of plasmid pAF1 insertion in strains CHP606, CHP616 and CHP617 are shown. In each case, the $5^{\prime}$ to $3^{\prime}$ orientation of the affected gene is left to right. Because of the cloning strategy (see Figure 1), only the sequence of the genome adjacent to the bla gene (conferring ampicillin-resistance) can be determined. The site of insertion is identified with respect to the start of translation of the affected gene. An additional $268 \mathrm{bp}$ of the his 3 gene is present in CHP616, possibly due to concatemerization and rearrangement of the plasmid DNA before insertion.

insert was very small, suggests that this is not a reliable strategy. Finally, when multiple E. coli transformants were obtained, we purified and analyzed plasmids from two or three individual transformants. In each case, plasmids obtained from the same rescue protocol were identical.

\section{Sequence Analyses of Rescued Plasmids}

Rescued plasmids were sequenced to identify the site of insertion (using primer BS250) and the site cut within the flanking genomic DNA (using the $\mathrm{T} 3$ promoter primer). As shown in Figure 1 , these plasmids contain only one of the two junctions between the genomic and plasmid DNA from the chromosomal insertion. Therefore, we cannot determine whether or not sequence from the genomic DNA is lost during plasmid integration. The sequencing results are summarized in Table 2 and presented as a schematic in Figure 2.

In CHP616, a small portion of the his 3 gene was found at the insertion junction, suggesting that a concatemer of plasmid DNA may have formed and rearranged before insertion into the genome. This observation indicates that tandem plasmid integrations could occur, complicating the rescue of flanking genomic DNA. Two of the three integrations were $5^{\prime}$ to the open reading frame and produced relatively weak mutant alleles of git5 (CHP606) and pkal (CHP617) as judged from the $\beta$ gal assays (not shown). Previous studies showed that git $5^{+}$, encoding the beta subunit of a herterotrimeric $\mathrm{G}$ protein required for adenylate cyclase activation, and $\mathrm{pkal}^{+}$(formerly git $6^{+}$), encoding the catalytic subunit of PKA, are required for $f b p l$ repression $(8,9$, 10; Landry, Pettit, Apolinario and Hoffman, unpublished). The third integration identified a novel gene that we determined to be git $3^{+}$by linkage and complementation tests with previously designated git3- mutants (data not shown; 8,9). We used the flanking DNA from the SacII-rescued plasmid to identify cosmid clones carrying the intact git $3^{+}$gene, which allowed us to clone and sequence git $3^{+}$(Welton and Hoffman, unpublished), thereby identifying the site of insertion relative to the 
open reading frame.

Sequence analysis from the BS250 primer also showed that only the 4 base NgoMIV overhang was lost from plasmid pAF1 in the CHP606 and CHP617 insertions, while one additional base pair of sequence was lost in the CHP616 insertion. This observation suggests that one could carry out the insertional mutagenesis with plasmid pAF1 linearized with any of the restriction enzymes that cut uniquely in the polylinker $5^{\prime}$ to the his $3^{+}$gene (KpnI, EcoO109I, ApaI, HincII, or SalI) without the danger of loss of his 3 promoter sequence during insertion. The potential advantage to varying the linearization site is that it may alter the profile of the insertion sites to generate different collections of mutants. Finally, the sequences of the genomic DNA at the three sites of integration do not indicate that plasmid insertion displays any strong sequence specificity (Table 2). The sites of insertion are all A-T rich, but this is generally true of yeast genomic DNA.

\section{CONCLUSIONS}

This study demonstrates our ability to generate mutations in several genes and to rapidly clone and identify the genes affected. We did not directly address the degree to which insertions occur randomly. However, the fact that the three insertions were in three different genes suggests that the site of insertion is not too restricted for use of this technique as a method of mutagenesis. Our ability to rescue the integrated plasmid using a variety of different restriction enzymes indicates that one is unlikely to obtain an integration that is resistant to rescue because of a paucity of restriction sites in the flanking sequence. Finally, two of three integrations were $5^{\prime}$ to the affected open reading frames and conferred a weak mutant phenotype relative to the commensurate gene disruptions, which suggests that this procedure may also be effective in the identification and cloning of essential genes.

\section{ACKNOWLEDGMENTS}

We thank Kathy Gould and Ryoma Ohi for plasmid pAF1 and his3-DI strains. We also thank Paul Young for advice and discussions regarding insertional mutagenesis in S. pombe and for sharing information on his work using the $\mathrm{ura}^{+}$gene. This work was supported by National Institutes of Health Grant No. GM46226 to C.S.H.

\section{REFERENCES}

1.Burke, J.D. and K.L. Gould. 1994. Molecular cloning and characterization of the Schizosaccharomyces pombe his 3 gene for use as a selectable marker. Mol. Gen. Genet. 242:169-176.

2.Carbon, J. and L. Clarke. 1990. Centromere structure and function in budding and fission yeasts. New Biol. 2:10-19.

3.Caspari, T. 1997. Onset of gluconate-H+ symport in Schizosaccharomyces pombe is regulated by the kinases Wis 1 and Pka1, and requires the gtil $^{+}$gene product. J. Cell Sci. 110: 2599-2608.

4.Dal Santo, P., B. Blanchard and C.S. Hoffman. 1996. The Schizosaccharomyces pombe pyp 1 protein tyrosine phosphatase negatively regulates nutrient monitoring pathways. J. Cell Sci. 109:1919-1925.

5.Gutz, H., H. Heslot, U. Leupold and N. Loprieno. 1974. Schizosaccharomyces pombe, $\mathrm{p}$.
395-446. In R.C. King (Ed.), Handbook of Genetics. Plenum Press, New York.

6.Heyer, W.D., M. Sipiczki and J. Kohli. 1986. Replicating plasmids in Schizosaccharomyces pombe: improvement of symmetric segregation by a new genetic element. Mol. Cell. Biol. 6:80-89.

7.Hoffman, C.S. and F. Winston. 1987. A tenminute DNA preparation from yeast efficiently releases autonomous plasmids for transformation of Escherichia coli. Gene 57:267-272.

8.Hoffman, C.S. and F. Winston. 1990. Isolation and characterization of mutants constitutive for expression of the fbpl gene of Schizosaccharomyces pombe. Genetics 124:807-816.

9.Hoffman, C.S. and F. Winston. 1991. Glucose repression of transcription of the Schizosaccharomyces pombe fbpl gene occurs by a cAMP signaling pathway. Genes Dev. 5:561-571.

10.Jin, M., M. Fujita, B.M. Culley, E. Apolinario, M. Yamamoto, K. Maundrell and C.S. Hoffman 1995. sckl, a high copy number suppressor of defects in the cAMP-dependent protein kinase pathway in fission yeast, encodes a protein homologous to the Saccharomyces cerevisiae SCH9 kinase. Genetics 140:457-467.

11.Nasim, A., P. Young and B.F. Johnson. 1989. Molecular Biology of the Fission Yeast. Academic Press, San Diego.

12.Ohi, R., A. Feoktistova and K.L. Gould. 1996. Construction of vectors and a genomic library for use with his3-deficient strains of Schizosaccharomyces pombe. Gene 174:315318.

13.Pringle, J.R., J.R. Broach and E.W. Jones. 1997. Molecular and Cellular Biology of the Yeast Saccharomyces. Vol. III. CSH Laboratory Press, Cold Spring Harbor, NY.

14.Rose, M.D., P. Novick, J.H. Thomas, D. Botstein and G.R. Fink. 1987. A Saccharomyces cerevisiae genomic plasmid bank based on a centromere-containing shuttle vector. Gene 60:237-243.

15.Sherman, F., G.R. Fink and C.W. Lawrence. 1978. Methods in Yeast Genetics: A Laboratory Manual. CSH Laboratory Press, Cold Spring Harbor, NY.

16.Wantanabe, Y., Y. Iino, K. Furuhata, C. Shimoda and M. Yamamoto. 1988. The Schizosaccharomyces pombe mei2 gene encoding a crucial molecule for commitment to meiosis is under the regulation of cAMP. EMBO J. 7:761-767.

Received 6 August 1999; accepted 20 October 1999.

Address correspondence to:

Dr. Charles S. Hoffman

Department of Biology

Boston College

Higgins Hall 315

Chestnut Hill, MA 02467, USA

Internet: hoffmacs@bc.edu 